\title{
A Case of Combined Sarcoidosis and Usual Interstitial Pneumonia
}

\author{
Kazunobu Tachibana ${ }^{1,2}$, Toru Arai ${ }^{1,2}$, Tomoko Kagawa ${ }^{2}$, Shojiro Minomo ${ }^{2}$, Masanori Akira ${ }^{3}$, \\ Masanori Kitaichi ${ }^{4}$ and Yoshikazu Inoue ${ }^{1}$
}

\begin{abstract}
Sarcoidosis is a systemic granulomatous disease of unknown etiology with characteristic pulmonary lesions, which are often distributed in the upper lung fields. We describe a unique case of sarcoidosis with lower lung field-dominant reticular shadows. Three years after the diagnosis of sarcoidosis based on histologic findings of the mediastinal lymph nodes and transbronchial lung biopsy specimens, the patient developed acute respiratory failure and died. The autopsy showed usual interstitial pneumonia (UIP), with honeycombing and superimposed diffuse alveolar damage of the lungs. The findings suggest that the patient had both sarcoidosis and UIP, and that the UIP later progressed to acute exacerbation.
\end{abstract}

Key words: sarcoidosis, usual interstitial pneumonia, acute exacerbation

(Intern Med 51: 1893-1897, 2012)

(DOI: 10.2169/internalmedicine.51.7394)

\section{Introduction}

Sarcoidosis is a systemic granulomatous disease of unknown etiology. Its radiologic characteristics include reticular opacities that are distributed predominantly in the upper lung fields (1). The radiologic finding of lower zonedominant reticular shadowing is encountered only rarely in sarcoidosis $(2,3)$; however, this pattern is one of the characteristics of idiopathic pulmonary fibrosis/usual interstitial pneumonia (IPF/UIP) (4). Some patients with IPF are known to experience an acute exacerbation following a period of chronic disease progression (5-8). We recently encountered a patient with both pulmonary sarcoidosis and UIP, who developed an acute exacerbation of the latter condition, leading to his death from acute respiratory failure. The patient's clinical course could not be explained solely by sarcoidosis, but mimicked IPF. Thus, this case may assist in understanding the potential for sarcoidosis and UIP comorbidity, as well as the pathogenesis of reticular opacities distributed predominantly in the lower lung fields in pa- tients with sarcoidosis.

\section{Case Report}

A 62-year-old man, an ex-smoker, was admitted to the Kinki-Chuo Chest Medical Center for the assessment of abnormal chest X-ray and computed tomography (CT) findings in 2007. He had worked as a truck driver for 14 years (from the age of 23 until the age of 37 years) and as a building demolition worker for the subsequent 25 years (from the age of 37 years to 62 years). He reported a four-month history of dry cough and worsening of dyspnea on effort. His only other relevant medical history was fatty liver, and hyperuricemia, which was treated with allopurinol. He had used a down quilt on the bed since age 32. Physical examination on admission revealed no palpable surface lymph nodes, no finger clubbing, and no skin rash; however, fine crackles were heard on chest auscultation. Peripheral blood findings on admission were as follows: WBC count, $5,900 / \mu \mathrm{L}$; lactate dehydrogenase $(\mathrm{LDH}), 255 \mathrm{IU} / \mathrm{L}$; and $\mathrm{C}$-reactive protein (CRP) $0.09 \mathrm{mg} / \mathrm{dL}$. The levels of Krebs von den Lungen-6

\footnotetext{
${ }^{1}$ Department of Diffuse Lung Diseases and Respiratory Failure, National Hospital Organization Kinki-Chuo Chest Medical Center, Japan, ${ }^{2}$ Department of Respiratory Medicine, National Hospital Organization Kinki-Chuo Chest Medical Center, Japan, ${ }^{3}$ Department of Radiology, National Hospital Organization Kinki-Chuo Chest Medical Center, Japan and ${ }^{4}$ Department of Pathology, National Hospital Organization Kinki-Chuo Chest Medical Center, Japan

Received for publication January 24, 2012; Accepted for publication April 2, 2012

Correspondence to Dr. Yoshikazu Inoue, giichi@kch.hosp.go.jp
} 

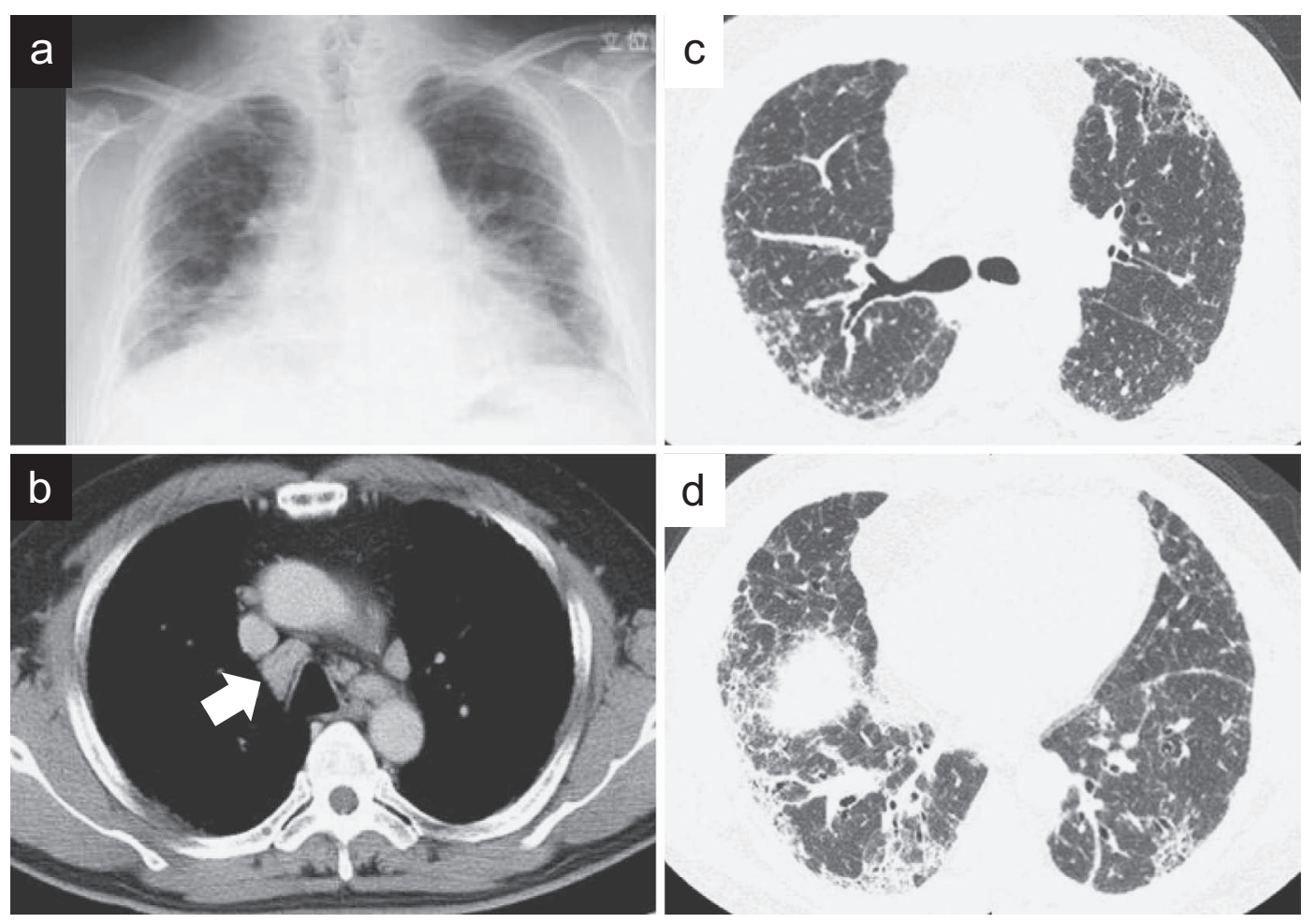

Figure 1. Chest radiograph (a) and CT scan (b-d) of the lung at the initial visit (2007), showing perilymphatic distributed small nodules, reticular opacities predominantly in the lower lung fields, and mediastinal lymphadenopathy (arrow).
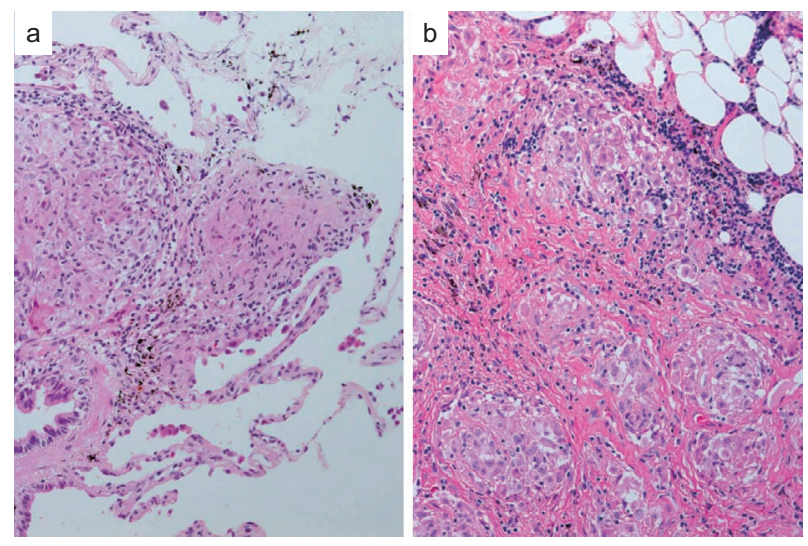

Figure 2. (a) The lung tissue of the right lower lobe (segment 8) was obtained by a transbronchoscopic biopsy in 2007. Epithelioid cell granulomas were formed in the wall of a respiratory bronchiole. Adjacent alveolar walls were relatively normal (Hematoxylin and Eosin staining, $\times 20$ ). (b) The mediastinal lymph node was obtained by a mediastinoscopic biopsy in 2007. Numerous epithelioid cell granulomas were formed in the lymph node with perigranulomatous hyalinous fibrotic changes (Hematoxylin and Eosin staining, $\times 20$ ). Based on the histologic evidence of the lung tissue and the mediastinal lymph node, the authors considered that the patient had sarcoidosis at the time of his initial visit in 2007.

(KL-6) and surfactant protein-D (SP-D) were increased (3,600 U/mL and $492 \mathrm{ng} / \mathrm{mL}$, respectively), and an elevated activity of both angiotensin-converting enzyme (ACE) (25.1
U/lL; normal range: 8.3-21.4 U/L) and lysozyme (10.6 $\mu \mathrm{g} /$ $\mathrm{mL}$; normal range: $5.0-10.2 \mu \mathrm{g} / \mathrm{mL}$ ) was observed. The patient tested negative for antinuclear antibody and antidouble-stranded DNA. Tuberculin reaction was also negative.

Chest radiography revealed diffuse reticular opacities in the outer and lower zones of the lungs along with elevation of the diaphragm (Fig. 1a). A high-resolution CT scan of the chest demonstrated reticular shadows in the subpleural area, traction bronchiectasis, small nodules in perilymphatic and random distribution, and enlargement of the mediastinal lymph nodes (lower paratracheal lymph nodes) (Fig. 1b-d). Bronchoscopy revealed normal bronchial mucosa. Bronchoalveolar lavage (BAL) was performed in the right $\mathrm{B}^{4} \mathrm{~b}$. The recovery rate of the bronchoalveolar lavage fluid (BALF) was $47 \%$, and the total cell count in the fluid was $3.12 \times 10^{5} / \mathrm{mL}$, with a breakdown of $45.8 \%$ macrophages, $16.3 \%$ lymphocytes, $11.7 \%$ neutrophils, and $26.2 \%$ eosinophils. The lymphocyte CD4/CD8 ratio was 7.41. No pathogen was detected in the BALF. Transbronchial biopsies of the right lung revealed noncaseating epithelioid cell granulomas in both $\mathrm{S}^{3}$ and $\mathrm{S}^{8}$ (Fig. 2a and data not shown). A mediastinoscopic biopsy of the mediastinal lymph nodes revealed numerous noncaseating epithelioid granulomas surrounded by hyalinous fibrotic changes (Fig. 2b). Based on these findings, a diagnosis of sarcoidosis involving the lungs and mediastinal lymph nodes was made. No sarcoidosis lesions were observed in other tissues including the heart, eyes and skin. At this time, the findings on chest X-ray and 


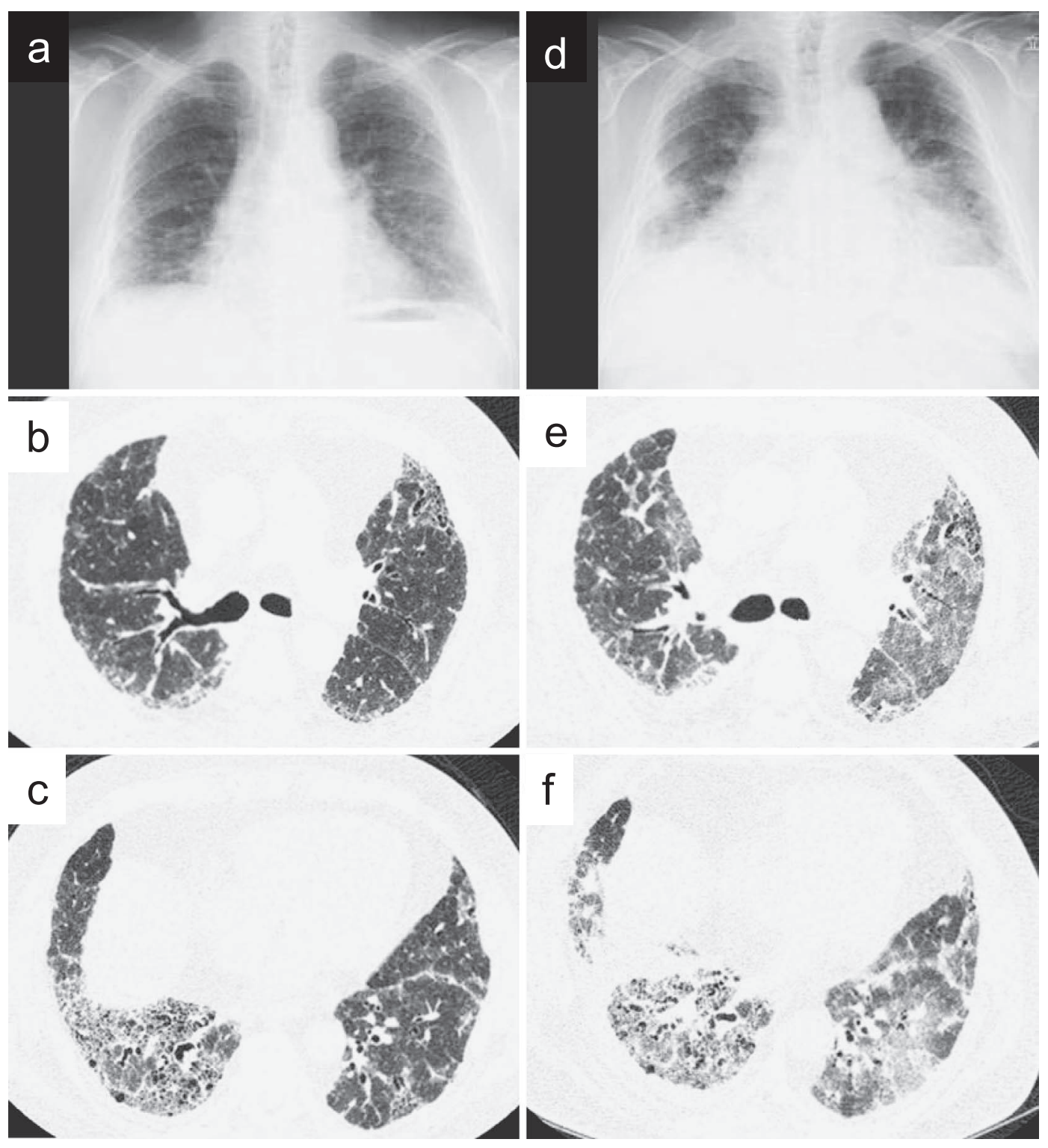

Figure 3. Chest radiograph (a) and CT scan (b, c) of the lung, 3 years after the initial visit (2007), showing progressed reticulation and volume loss. Chest radiograph (d) and CT scan (e, f) of the lung at the time of acute respiratory failure, showing diffuse ground glass opacities superimposed on reticular opacities.

on $\mathrm{CT}$ of the bilateral fibrotic lung lesions, predominantly in the lower lung zones, were considered to be due to the fibrotic lesions of sarcoidosis itself.

As treatment, $35 \mathrm{mg}$ per day of oral prednisolone was started and then tapered. The patient's cough and dyspnea on exertion improved in two weeks, and an increase in vital capacity (VC) and diffusing capacity of the lung for carbon monoxide (DLco) were observed after a month, along with a slight improvement of small nodules in the lung detected on high-resolution CT and a decrease in the level of serum ACE. However, 20 months later, the patient experienced increasing shortness of breath, and chest imaging showed worsening of fibrotic shadows. Red-brown papules also appeared on the patient's lower legs, and these were diagnosed as cutaneous sarcoidosis by a dermatologist. These findings were presumed to indicate progression of the patient's sar- coidosis, and he was therefore treated with $35 \mathrm{mg}$ per day of oral prednisolone, combined with $100 \mathrm{mg}$ per day of azathioprine for the first seven months, switched to $100 \mathrm{mg}$ of cyclosporine for the latter four months of this period, and $50 \mathrm{mg}$ per week of etanercept for the final three months. However, this treatment was unsuccessful in preventing progression of the disease (Fig. 3a-c). Three years and two months after the initial diagnosis of sarcoidosis, a mass shadow in the right lung $\left(\mathrm{S}^{6}\right)$ was noted on chest CT. A CTguided needle biopsy revealed adenocarcinoma with thyroid transcription factor-1 (TTF-1)-positivity, suggestive of primary lung cancer.

One and a half months later, just before staging of the lung cancer, the patient presented with sudden onset of dyspnea and hemosputum. Chest X-ray and CT showed diffuse ground glass opacities (Fig. 3d-f) in both lungs. The 


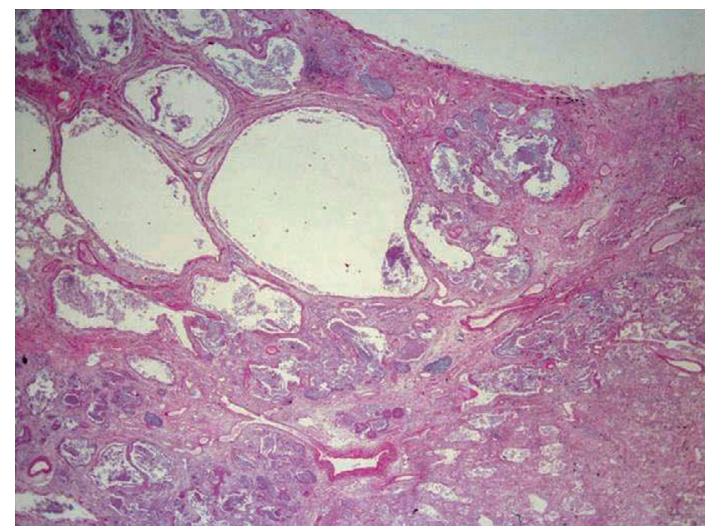

Figure 4. The lung tissue was obtained from the left lower lobe (segment 8) by an autopsy in 2010 . The subpleural region (upper and left) showed cystic changes measuring 0.7-4 $\mathrm{mm}$ in diameter surrounded with fibrotic lesions (honeycombing). The inner lung parenchyma (lower and left) showed diffuse interstitial fibrotic thickening with temporal homogeneity. The authors considered the lung tissue as showing usual interstitial pneumonia (UIP) superimposed with fibrotic stage of diffuse alveolar damage (DAD). No granulomas were found (Hematoxylin and Eosin staining, $\times 1$ ).

level of serum ACE remained normal. Treatment with highdose corticosteroids, cyclophosphamide, antibiotics, and invasive positive-pressure ventilation was ineffective, and the patient died from respiratory failure 40 months after the initial diagnosis of sarcoidosis (nine days after the commencement of high-dose corticosteroid treatment).

An autopsy was performed, focusing on the thoracic and abdominal organs, which revealed multiple bilateral parietal pleural plaques (right: $50 \times 50 \mathrm{~mm}, 20 \times 20 \mathrm{~mm}$; left: $45 \times 20$ $\mathrm{mm}, 40 \times 12 \mathrm{~mm}, 30 \times 15 \mathrm{~mm}, 40 \times 12 \mathrm{~mm})$. Eighty-three paraffin blocks were prepared for histopathologic diagnosis, including 14 from the right lung, 13 from the left lung, 4 from the mediastinal lymph nodes, and 2 each from the right and left parietal pleural plaque regions. We diagnosed the lung tissues as showing an UIP pattern, with honeycombing and superimposed diffuse alveolar damage (DAD) (Fig. 4). Asbestos bodies were not detected histologically, even after Prussian blue iron staining of the lung specimens. Therefore, bilateral lung tissues from the upper and lower lobes (right $S^{2}$, right $S^{10}$, left $S^{1+2}$, and left $S^{10}$ ) were measured for asbestos bodies. A total of 328 asbestos bodies per gram of dry weight of lung tissue were detected in the left lower lobe $\left(\mathrm{S}^{10}\right)$. The Helsinki Criteria for significant or occupational asbestos exposure is $>1,000$ asbestos bodies per gram of dry weight of lung tissue (9). Poorly differentiated adenocarcinoma $(20 \times 19 \times 15 \mathrm{~mm}$ in size $)$ was observed in the right lung $\left(S^{6}\right)$, and metastatic carcinoma was noted in the right pleura, liver, and the pulmonary hilar and mediastinal lymph nodes. However, no granulomas were found in the examined tissues.

Based on these findings, we concluded that the patient suffered from sarcoidosis and UIP during the observation period, and subsequently succumbed to an acute exacerbation 3 years later. Taken together, we propose that our findings more likely indicate that the UIP and bilateral parietal pleural plaques had been present concurrently with the sarcoidosis at the time of diagnosis of the latter, rather than a scenario where the sarcoid granulomas underwent progressive pulmonary fibrosis.

\section{Discussion}

We have described a patient with evidence of both sarcoidosis and UIP characterized by lower lung fieldpredominant pulmonary fibrosis, who developed an acute exacerbation 3 years after the initial diagnosis of sarcoidosis.

The patient died from acute respiratory failure. It is possible that this was acute respiratory failure due to sarcoidosis (10-13), in which severe alveolitis with inflammatory or organized exudates in some alveolar spaces and noncaseating epithelioid granulomas are observed (14). However, this is unlikely in the present case for a number of reasons: (i) the autopsy revealed no granulomas in the lungs and lymph nodes, but rather showed diffuse alveolar damage superimposed on UIP; (ii) the level of serum ACE remained in the normal range; and, (iii) in cases of acute respiratory failure due to sarcoidosis, granulomas are usually observed in the lung despite having been treated with corticosteroids (14). As a result, we consider it more likely that this case was an acute exacerbation that developed during the course of UIP rather than acute respiratory failure due to sarcoidosis.

Radiographic and histologic findings suggest that the patient had both sarcoidosis and UIP. There are two possibilities: that the sarcoidosis and UIP existed concurrently; or that the fibrotic lesions developed as a fibrotic stage of sarcoidosis. For instance, Nobata et al. have reported a case of pulmonary sarcoidosis with UIP distributed predominantly in the lower lung fields. They noted the possibility of a fibrotic stage of pulmonary sarcoidosis (3) because the fibrosis was distributed along the bronchovascular bundles, which is a feature of the fibrotic stage of pulmonary sarcoidosis (15). In the present case, however, it is thought that the acute exacerbation developed during the chronic course of UIP as mentioned above. This theory is supported by the fact that the autopsy revealed no concentric fibrosis or concentric lamellar calcifications (Schumann bodies), which are characteristic of the fibrotic stage of sarcoidosis (16). Moreover, fibrotic lesions caused by sarcoidosis are predominantly distributed in the upper lung fields; $68 \%$ of sarcoidosis cases had fibrotic lesions predominantly in the upper lung fields, with no more than $4.5 \%$ of cases in the lower lung fields (2). As noted, in the present case, the UIP lesions were predominantly distributed in the lower lung fields. Taken together, these findings lead us to suggest that the present patient was concurrently suffering from both sarcoidosis and UIP.

During the course of observation, the patient had been 
suspected of having chronic hypersensitivity pneumonia because of his history of using a down quilt on the bed. However, cessation of the use of down quilt neither improved the patient's condition nor stopped the disease progression. In addition, numerous well-developed granulomas were observed in the mediastinal lymph nodes (Fig. 2b). These findings therefore did not support the likelihood of chronic hypersensitivity pneumonia.

The autopsy showed parietal pleural plaques bilaterally, suggesting asbestos exposure. In general, asbestosis (a kind of lung fibrosis) is associated with a high level of exposure to asbestos (9). Unlike the present case, patients with asbestosis generally have a significant asbestos fiber burden and more extensive pleural plaques, which may be detected on chest CT (9). We believe that this patient probably had UIP and associated bilateral parietal pleural plaques as a result of his long duration of low-dose exposure to asbestos fibers, although progression of asbestosis is generally slow, the tenyear survival rate being around $70 \%(17,18)$. This raises the possibility that the low-dose asbestos exposure might have caused sarcoid-like reaction as reported in cases of highdose asbestos exposure $(19,20)$.

In summary, we have presented here a case of combined sarcoidosis and UIP with an acute exacerbation. This case is important in highlighting the possibility that sarcoidosis may develop after long-standing pulmonary fibrosis. Additional cases of lower lung field-predominant sarcoidosis should be studied in the future in order to further develop our understanding of this condition.

The authors state that they have no Conflict of Interest (COI).

\section{Acknowledgement}

The authors are grateful to Mr. Tomoaki Teramoto for performing the measurement of asbestos bodies from the lung tissues. This report was partially supported by a grant to the Diffuse Lung Diseases Research Group from the Ministry of Health, Labour and Welfare, Japan, and a grant from National Hospital Organization Respiratory Network.

\section{References}

1. Primack SL, Hartman TE, Hansell DM, Muller NL. End-stage lung disease: CT findings in 61 patients. Radiology 189: 681-686, 1993.

2. Brauner MW, Grenier P, Mompoint D, Lenoir S, de Cremoux H. Pulmonary sarcoidosis: evaluation with high-resolution CT. Radiology 172: 467-471, 1989.

3. Nobata K, Kasai T, Fujimura M, et al. Pulmonary sarcoidosis with usual interstitial pneumonia distributed predominantly in the lower lung fields. Intern Med 45: 359-362, 2006.

4. Staples CA, Muller NL, Vedal S, Abboud R, Ostrow D, Miller RR. Usual interstitial pneumonia: correlation of CT with clinical, functional, and radiologic findings. Radiology 162: 377-381, 1987.

5. Akira M, Hamada H, Sakatani M, Kobayashi C, Nishioka M, Yamamoto S. CT findings during phase of accelerated deterioration in patients with idiopathic pulmonary fibrosis. AJR Am J Roentgenol 168: 79-83, 1997.

6. Collard HR, Moore BB, Flaherty KR, et al. Acute exacerbations of idiopathic pulmonary fibrosis. Am J Respir Crit Care Med 176: 636-643, 2007.

7. Kondoh Y, Taniguchi H, Kawabata Y, Yokoi T, Suzuki K, Takagi $\mathrm{K}$. Acute exacerbation in idiopathic pulmonary fibrosis. Analysis of clinical and pathologic findings in three cases. Chest 103: 1808-1812, 1993.

8. Tachibana K, Inoue Y, Nishiyama A, et al. Polymyxin-B hemoperfusion for acute exacerbation of idiopathic pulmonary fibrosis: serum IL-7 as a prognostic marker. Sarcoidosis Vasc Diffuse Lung Dis 28: 113-122, 2011.

9. Asbestos, asbestosis, and cancer: the Helsinki criteria for diagnosis and attribution. Scand J Work Environ Health 23: 311-316, 1997.

10. Gupta D, Agarwal R, Paul AS, Joshi K. Acute hypoxemic respiratory failure in sarcoidosis: case report and review of the literature. Respir Care 56: 1849-1852, 2011.

11. Leiba A, Apter S, Leiba M, Thaler M, Grossman E. Acute respiratory failure in a patient with sarcoidosis and immunodeficiency: an unusual presentation and a complicated course. Lung 182: 7377, 2004

12. Pugazhenthi M. Sarcoidosis presenting as acute respiratory failure. South Med J 98: 265, 2005.

13. Sabbagh F, Gibbs C, Efferen LS. Pulmonary sarcoidosis and the acute respiratory distress syndrome (ARDS). Thorax 57: 655-656, 2002.

14. Shibata S, Saito K, Ishiwata N, Ieki R. A case of sarcoidosis presenting with high fever and rash progressing to acute respiratory failure. Nihon Kokyuki Gakkai Zasshi 45: 691-697, 2007 (in Japanese, Abstract in English).

15. Padley SP, Padhani AR, Nicholson A, Hansell DM. Pulmonary sarcoidosis mimicking cryptogenic fibrosing alveolitis on CT. Clin Radiol 51: 807-810, 1996.

16. Fraser RS, Muller NL, Colman N, Pare PD. Fraser and Pare's Diagnosis of Diseases of the Chest. 4th ed. W.B. Saunders, Philadelphia, 1999.

17. Coutts II, Gilson JC, Kerr IH, Parkes WR, Turner-Warwick M. Mortality in cases of asbestosis diagnosed by a pneumoconiosis medical panel. Thorax 42: 111-116, 1987.

18. Markowitz SB, Morabia A, Lilis R, Miller A, Nicholson WJ, Levin S. Clinical predictors of mortality from asbestosis in the North American Insulator Cohort, 1981 to 1991. Am J Respir Crit Care Med 156: 101-108, 1997.

19. Kido M, Kajiki A, Hiraoka K, Horie A. Sarcoid reaction observed in a worker with a history of asbestos exposure. J UOEH 12: 355$360,1990$.

20. Granel B, Serratrice J, Disdier P, et al. Sarcoid-like pulmonary lesions during asbestosis. A case report. Sarcoidosis Vasc Diffuse Lung Dis 17: 297, 2000.

(C) 2012 The Japanese Society of Internal Medicine http://www.naika.or.jp/imindex.html 